\title{
Morphological Analyses on Retinal Glial Responses to Glaucomatous Injury Evoked by Venous Cauterization
}

*Correspondence to:

Oh SJ,

Tel: +82-2-2258-7259

Fax: +82-2-536-3110

E-mail: sujaoh@catholic.ac.kr

Received March 20, 2014

Revised March 27, 2014

Accepted March 27, 2014

\author{
Ji-Yeon Lee, Ji-Man Shin, Myung-Hoon Chun, Su-Ja Oh* \\ Department of Anatomy, College of Medicine, The Catholic University of Korea, Seoul 137-701, Korea
}

\begin{abstract}
Retinal glial responses to hypertensive glaucomatous injury were spatiotemporally surveyed. Retinas as a whole or vertical sections were processed for anti-glial fibrillary acidic protein (GFAP), anti-Iba1, anti-nerve growth factor (NGF), and anti-tumor necrosis factor (TNF)- $\alpha$ immunohistochemistry for confocal microscopic analyses. The optic nerve head of paired controls was processed for electron microscopy. GFAP positive astrocytes appeared in the nerve fiber layer in the glaucomatous and control retinas, changing from fine protoplasmic to stout fibrous parallel to glaucomatous duration. Ibal positive microglia appeared in both retinas, and enormous reaction appeared at the latest glaucomatous. Müller reaction detected by GFAP reactivity expanded from the end feet to whole profile following to duration in the glaucomatous. NGF reactivity expended from the end feet to the proximal radial processes of the Müller cells in both retinas according to glaucomatous duration. TNF- $\alpha$ immunoreactivity in the nerve fiber layer was stronger in both the glaucomatous and controls than in the normal, and exceptionally at the latest glaucomatous was even lower than the normal. The astrocytes in the optic nerve head are interconnected with each other via gap junction. These results demonstrate that astrocyte reaction propagates to the contralateral via physical links, and TNF- $\alpha$ is correlated with NGF production for neuroprotection in response to hypertensive glaucomatous injury.
\end{abstract}

Key Words: Glial reaction, Glaucomatous injury, Growth factor, Confocal microscopy, Electron microscopy

\section{INTRODUCTION}

The retina is a primary visual organ as converting absorbed photons from light into neural signals and processing the visual information prior to transport to the higher visual center (Rodrieck, 1998). It contains several classes of neuronal cells and supportive glial cells as a part of the central nervous system (CNS). The retinal neuronal cells are well organized into three layers of neuronal somatas such as the outer nuclear layer, the inner nuclear layer and the ganglion cell layer (GCL), and three layers of their processes such as the outer plexiform layer, the inner plexiform layer and the nerve fiber layer (NFL). Three kinds of glial cells are usually resident in the mammalian retina (Bringmann et al., 2006). The first one,
Müller glial cell is distributed throughout the whole depth and area of the retina and thus also called as Müller radial glia. The second is astrocytes, which are located in the NFL and the GCL close to retinal blood vessels (Stone \& Dreher, 1987). The last is microglia, which are normally restricted into the NFL and the GCL showing ramified morphology. The first and second types together are called as macroglia in contrast to microglia. The macroglia are generally known to play a supportive role constituting blood-retinal barrier and involving in extra-neuronal homeostasis (Bauer et al., 2005; Swanson, 2005; Bringmann et al., 2006). The microglia has a phagocytic and immune function (Bringmann et al., 2006).

The macroglia become activated or reactive in response to a variety of pathogenic stimuli, and these phenomena were

This study was supported by the Ministy of Knowledge Economy, Republic of Korea (10030064).

@ This is an open-access article distributed under the terms of the Creative Commons Attribution Non-Commercial License (http://creativecommons.org/licenses/by-nc/3.0) which permits unrestricted noncommercial use, distribution, and reproduction in any medium, provided the original work is properly cited.

Copyrights @ 2014 by Korean Society of Microscopy 
called glial reaction or gliosis. Glial reaction is quite different according to type of injuries or stimuli. A general cellular marker for glial reaction is the upregulation of a member of the intermediate filament protein family, glial fibrillary acidic protein (GFAP) (Bignami \& Dahl, 1979; Bringmann \& Reichenbach, 2001). Increase of GFAP expression was reported in various retinal diseases such as glaucoma (Francke et al., 1997; Tezel et al., 2003), ischemia-reperfusion (Akiyama et al., 2002; Eichler et al., 2004; Pannicke et al., 2004), and diabetic retinopathy (Hammes et al., 1995; Rungger-Brändle et al., 2000). Glial cells were known to additionally produce a variety of cytokines including growth factor and tumor necrosis factor family responding to injuries or stimuli (Aloisi, 2001; Delgado et al., 2003; Lee et al., 2012). These cytokines were documented to be involved in a variety of functions, including neuroprotection, tissue repair, glia activation, and tissue damage in response to numerous stimuli in the CNS. Glaucoma is defined as an optic neuropathy characterized by the accelerated death of retinal ganglion cells and their axons, and its prevalence increases markedly with aging (Quigley et al., 1995; Quigley \& Broman, 2006). It has traditionally been considered a disease of raised intraocular pressure (IOP) however the increase in its prevalence with aging does not correspond to increase in IOP (Varma et al., 2004). Furthermore, normotensive glaucoma patients are recently increased. These circumstances may provide a motive for intensive investigation on glial responses to glaucomatous injury.

Based on these findings, we aimed to investigate spatiotemporal spread of macroglial and microglial responses and their correlation in glaucoma for better understanding on the mechanism of glaucomatous neurodegeneration. For this experiment, a unilateral hypertensive glaucomatous injury model has been taken and retinal glial responses were evaluated by confocal microscopy using immunohistochemistry and partially by electron microscopy.

\section{MATERIALS AND METHODS}

\footnotetext{
Animals

Young adult Sprague-Dawley rats of 8 weeks age were used for the present study. All experimental procedures were submitted to the regulation of the Catholic Ethics Committee of the Catholic University of Korea, which conforms to the US National Institute of Health (NIH) guidelines for the Care and Use of Laboratory Animals (NIH Publications No. 8023) revised in 1996. The animals were cared under a 12 hours light/dark cycle, with standard chow and water ad libitum. This study was performed from January to December 2012 at College of Medicine, The Catholic University of Korea in Seoul, Korea.
}

\section{Glaucoma Injury Model Establishment}

Glaucomatous injury in this study was made by elevation of IOP via venous drainage blockade. Animals were anesthetized by an intraperitoneal injection of $50 \mathrm{mg} / \mathrm{kg}$ body weight tiletamine plus zolazepam (Zoletil; Virbac, France) and $15 \mathrm{mg} / \mathrm{kg}$ body weight xylazine hydrochloride (Rompun; Bayer, Germany). Proparacaine of $0.5 \%$ concentration was topically eye dropped to numb the cornea. To elevate the IOP, three episcleral veins in the right side eyeball were electrical cauterized under a surgical microscope (Olympus, Japan). A small conjunctival incision was made in each quadrant at the limbus, and the extraocular muscles were isolated. Among four major limbal draining veins, two dorsal and one temporal episcleral veins were cauterized with a 30-gauge tip using a cautery (Bovie Medical Co., USA). After cauterization, the incision was sutured with nylon 9-0 and disinfected with povidon. Animals with cauterization were grouped and cared for 1 week, and 4 and 8 weeks after cauterization. IOP was measured with a calibrated tonometer (Tono-Pen; Mentor $\mathrm{O} \& \mathrm{O}, \mathrm{USA})$ in the glaucomatous and their contra-lateral control eyeballs every week after cauterization.

\section{Tissue Preparation}

After checking the final IOP, the eyeballs including the optic nerve head at each experimental time point were enucleated under anesthesia with $4 \%$ chloral hydrate. The animals were killed with an overdose of chloral hydrate. The eyeballs were then encircled cut along the posterior margin of the ora serrata and their posterior halves were immersed in $4 \%$ paraformaldehyde in $0.1 \mathrm{~mol} / \mathrm{L}$ phosphate buffer, $\mathrm{pH} 7.4(\mathrm{~PB})$. The retinas were dissected out from posterior halves as a whole and immersed in the same fixative for 2 hours. After fixation, the retinal tissues were transferred to $30 \%$ sucrose solution in $\mathrm{PB}$ and kept overnight at refrigerator for preservation. The retinas were then quick frozen in liquid nitrogen and then stored at $-70^{\circ} \mathrm{C}$. For double immunohistochemistry, the retinas were processed as a whole. For single immunohistochemistry, the central portion of the superior quadrant in the whole retinas was trimmed into small pieces and rinsed with 0.01 $\mathrm{mol} / \mathrm{L}$ phosphate buffered saline in $\mathrm{pH} 7.4$ (PBS). After thorough rinsing, retinal pieces were dehydrated, embedded in wax and cut into $5 \mu \mathrm{m}$ thick vertical sections. The vertical retinal sections were mounted on albumin-coated slide glasses. For electron microscopy, the optic nerve heads from enucleated eyes of contra-lateral control to glaucomatous injured eyes were longitudinally bisected from the center of the optic disc to the center of the optic nerve.

\section{Immunohistochemistry}

To trace macro- and micro-glial response, whole-mount retinas were conducted with double immunofluorescent using monoclonal anti-GFAP antibody (diluted in 1:2000; 
Chemicon, USA) for macroglial cells and polyclonal antiIba1 antibody (diluted 1:500, Wako Pure Chemical Industries, Japan) for microglial cells. The retinas were previously treated to $10 \%$ normal donkey serum (NDS) (Jackson ImmunoResearch, USA) in PBS with $1 \%$ Triton X-100 (Sigma-Aldrich, USA) overnight at room temperature to mask the nonspecific binding sites in tissue. The retinas were then incubated in primary antibodies for 3 days at refrigerator, and rinsed with PBS for 3 times of 15 minutes. After thorough rinsing, the tissues were incubated with the appropriate secondary antibodies for 2 days at refrigerator, and rinsed with PBS for 45 minutes. The secondary antibodies used here were Alexa Fluor 488-conjugated antirabbit immunoglobulin $\mathrm{G}(\mathrm{IgG})$ antibody (diluted in 1:300; Life technologies, USA) and Cy3- conjugated anti-mouse IgG antibody (diluted in 1:2,000; Jackson ImmunoResearch).

For astrocyte and Müller glial responses, anti-GFAP immunohistochemistry was processed in the retinal sections using the avidin-biotin-peroxidase method (Hsu et al., 1981). After blocking the nonspecific binding sites by NDS treatment, the sections were incubated with monoclonal anti-GFAP antibody (Chemicon), washed in PBS for 45 minutes. The secondary antibody conjugated with biotin (Vector Laboratories, USA) was treated to these sections for 12 hours in refrigerator. The retinal sections were rinsed in two changes of PBS and three changes of $0.05 \mathrm{~mol} / \mathrm{L}$ Tris- $\mathrm{HCl}$ buffer, $\mathrm{pH}$ 7.6 (TB) for 5 minutes each, and then processed for color reaction in $0.05 \% 3,3^{\prime}$-diaminobenzidine tetrahydrochloride in TB for 10 minutes. Hydrogen peroxide was added to the incubation medium to a final concentration of $0.01 \%$. The reaction was terminated with several washes of $\mathrm{TB}$ and $\mathrm{PB}$ after proper color determination. The result was analyzed by a light microscope equipped with differential interference camera apparatus.

To investigate functional activation accompanying with glial reaction, the changes in the expression levels of nerve growth factor (NGF) and tumor necrosis factor (TNF)- $\alpha$ were evaluated in the retinal sections with immunohistochemistry. After blocking the nonspecific binding sites treated with NDS, the retinal sections were incubated with primary antibodies overnight in refrigerator and then rinsed with PBS for 45 minutes. The secondary antibody was Alexa Fluor 488-conjugated anti-rabbit IgG, applied to the sections for 2 hours, and then the sections were rinsed again with PBS for 45 minutes. The primary antibodies used here were polyclonal anti NGF- $\beta$ antibody (diluted in 1:200; Chemicon) and polyclonal anti-TNF- $\alpha$ antibody (diluted in 1:5,000; Millipore, USA).

\section{Confocal Microscopic Observation}

All retinal preparations processed for immunofluorescent staining were evaluated by confocal laser scanning microscopy
(LSM 510 Meta; Zeiss, Germany). Fluorescent images were captured with green laser (excitation $488 \mathrm{~nm}$, emission 490 555 nm) and red laser (excitation $555 \mathrm{~nm}$, emission $505 \sim 600 \mathrm{~nm}$ ) at 1,000 magnification power.

\section{Electron Microscopy}

Bisected optic nerve heads of the glaucomatous control were fixed in $4 \%$ paraformaldehyde- $1 \%$ glutaraldehyde solution in $\mathrm{PB}$ for 3 hours at refrigerator, thorough washed in $\mathrm{PB}$, and post-fixed in $1 \%$ osmium tetroxide in PB for 2 hours. Fixation processed tissue blocks were dehydrated with upgraded alcohol series, substituted to acetone and xylene and embedded in Poly/Bed 812 (Polysciences, USA) mixture. After polymerization of synthetic resin curing at $60^{\circ} \mathrm{C}$ for 3 days, one micrometer thick semi-thin sections were made from these blocks. Well preserved areas from these sections were chosen, and the blocks were trimmed into smaller and then sectioned into 70 80 nm thick sections. Ultrathin sections were mounted on Formvar coated one-hole grids, stained with uranyl acetate and lead citrate and examined using an electron microscope (JEM 1200 EX; Jeol, Japan).

\section{RESULTS}

The IOP after episcleral vein cauterization was successfully elevated and sustained throughout the experimental periods. The IOP in the glaucomatous injured eyes was measured about two-fold increase to the normal level at 1 week after venous cauterization and then maintained afterwards. That in the control eyes was recorded to the normal level (Fig. 1).

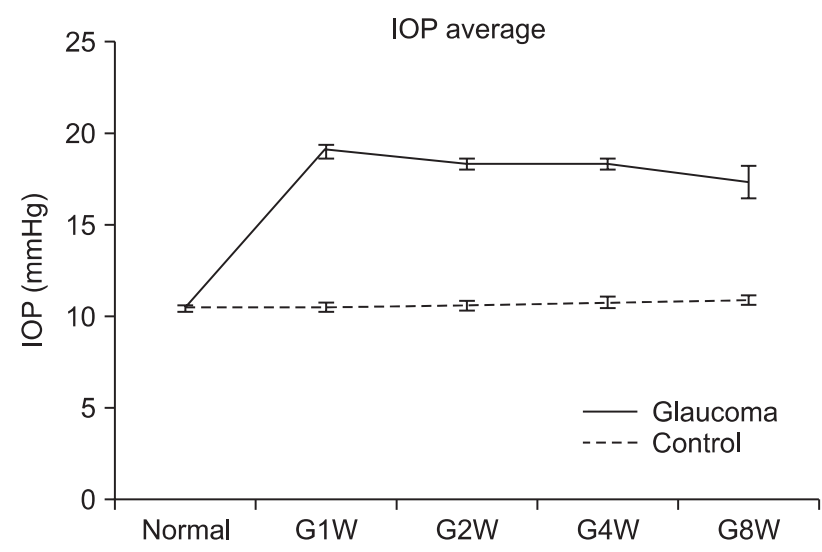

Fig. 1. IOP changes in the rat eyes after venous cauterized operation in the right side eye. IOP was assessed at normal retina and at 1, 2, 4, and 8 weeks glaucomatous retinas (G1W, G2W, G4W, and G8W). The solid line shows IOP changes in the glaucomatous eyes and the broken line, those in their contralateral control eyes. IOP was abruptly increased at G1W and sustained to G8W. In the contralateral control retinas of glaucoma, IOP shows no large change. Data are presented as mean \pm standard deviation. IOP, intraocular pressure. 


\section{Glial Fibrillary Acidic Protein and Iba1 Immunoreactivities}

In whole-mount retinas of the normal, GFAP and Ibal immunoreactivity appeared in star-shaped astrocytes and in ramified microglial cells along the blood vessels, respectively, and these glial cells were distributed mainly in the NFL and the GCL (Fig. 2A). GFAP immunoreactive astrocytes signaled with red color were changed in the shape of cytoplasmic process from a hundred fine fibrillar networking to stout coarse fibrous parallel to time lapse of the glaucomatous (Fig. $2 \mathrm{E}-\mathrm{G})$. With the same manner in the glaucomatous retinas, the astrocytes in whole-mount control retinas were also changed both in shape and in the immunoreactivity (Fig. 2BD). Iba1 immunoreactive microglial cells signaled with green color were increased in both shape and cell number in the glaucomatous retinas along the glaucomatous duration (Fig. $2 \mathrm{E}-\mathrm{G}$ ), whereas those cells in the paired control retinas were showed nearly no changes in shape and in cell number (Fig. 2B-D).

In vertical section preparation of the normal retina, three nuclear layers such as the outer nuclear layer, the inner nuclear layer and the GCL, and three nerve processes layers such as the outer plexiform layer, the inner plexiform layer and the NFL. GFAP immunoreactivity in the section of the normal retina appeared in the astrocytes and the end feet of Müller glial cells distributed in the NFL (Fig. 3A). In the glaucomatous retinal sections (Fig. 3E-G), GFAP immunoreactivity was extended into the proximal radial processes of Müller cells in the inner plexiform layer at 1 week (Fig. 3E), and even into the distal radial processes of Müller cells in the outer plexiform and nuclear layers at 4 weeks (Fig. 3F) and 8 weeks (Fig. 3G). In the control retinal sections (Fig. 3B-D), GFAP immunoreactivity was largely restricted into the astrocytes and into the end feet and proximal part of the proximal radial processes of Müller cells. The GFAP immunoreactivity in the control retinas was increased compared to the normal retina.

\section{Nerve Growth Factor and Tumor Necrosis Factor- $\alpha$ Immunoreactivities}

NGF immunoreactivity appeared faintly in the end feet and proximal part of the proximal radial processes of Müller glial cells in the sectional preparation of the normal retina (Fig. $4 \mathrm{~A}$ ). In the glaucomatous retina section preparations (Fig. 4E-G), NGF immunoreactivity appeared in the Müller end feet and expanded into proximal radial processes in parallel to time lapse. In the control retina preparations (Fig. 4B-D), NGF reactivity was also increased in the Müller end feet and proximal radial processes compared to the normal.

TNF- $\alpha$ immunoreactivity appeared faintly in the NFL and the GCL of the normal retinal section (Fig. 5A). In the glaucomatous retina section preparations (Fig. $5 \mathrm{E}-\mathrm{G}$ ), TNF- $\alpha$ reactivity was increased at 1 week (Fig. 5E) and 4 weeks (Fig.
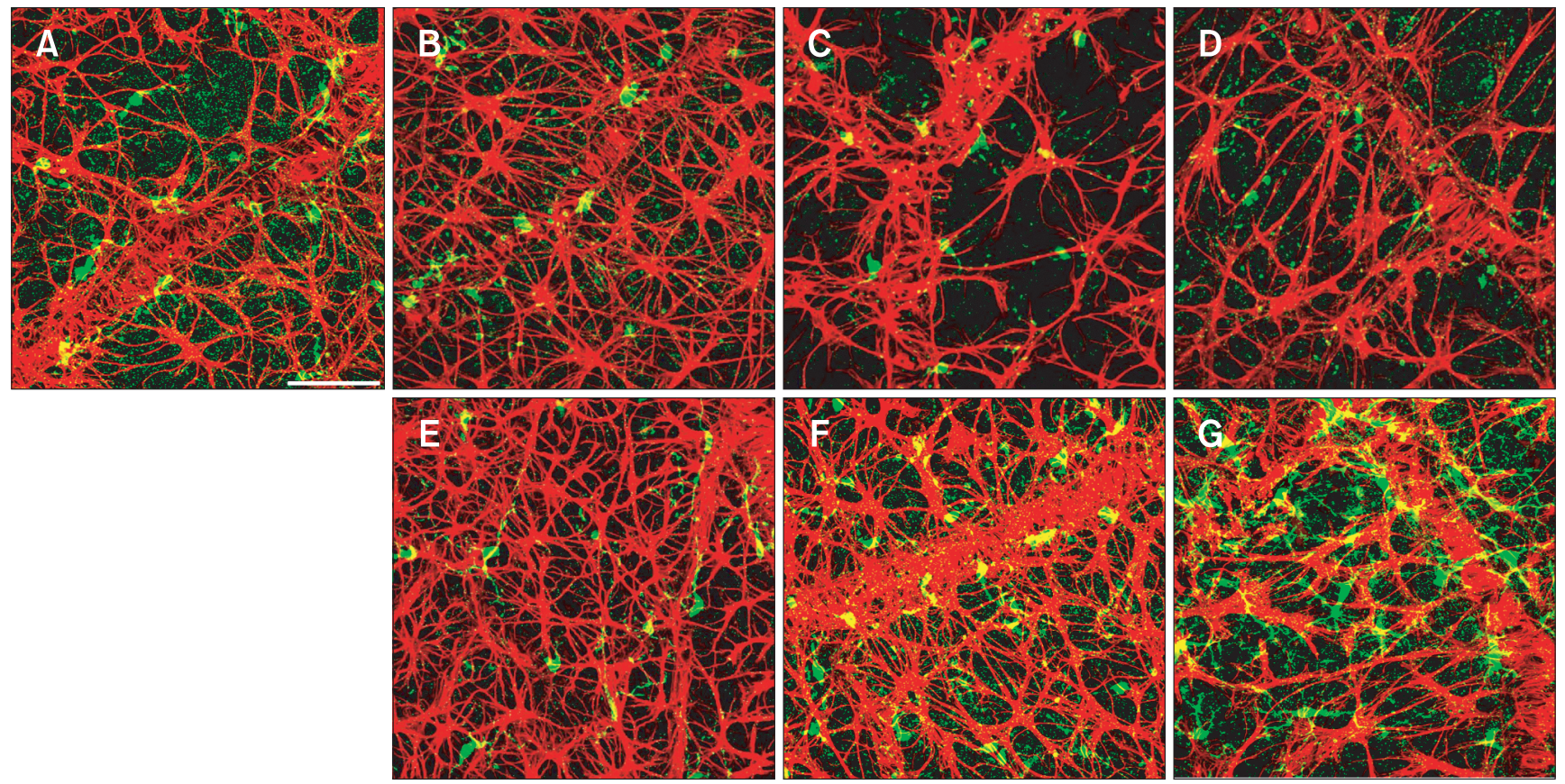

Fig. 2. Confocal microscopic views of whole-mounted retinas processed for anti-GFAP (red) and anti-Ibal (green) double immunofluorescent staining Focus is on the nerve fiber layer of normal retina (A), of contralateral control retinas (B-D) at 1 week (B), 4 weeks (C), and 8 weeks (D) glaucoma, and of ipsilateral glaucomatous retinas (E-G) at 1 week (E), 4 weeks (F), and 8 weeks (G). GFAP positive glial fiber network became stronger and heavier in parallel to increase of glaucoma duration. Ibal positive microglia was also increased in cell number and in reactivity according to glaucoma durations. Scale bar $=50 \mu \mathrm{m}$. GFAP, glial fibrillary acidic protein. 

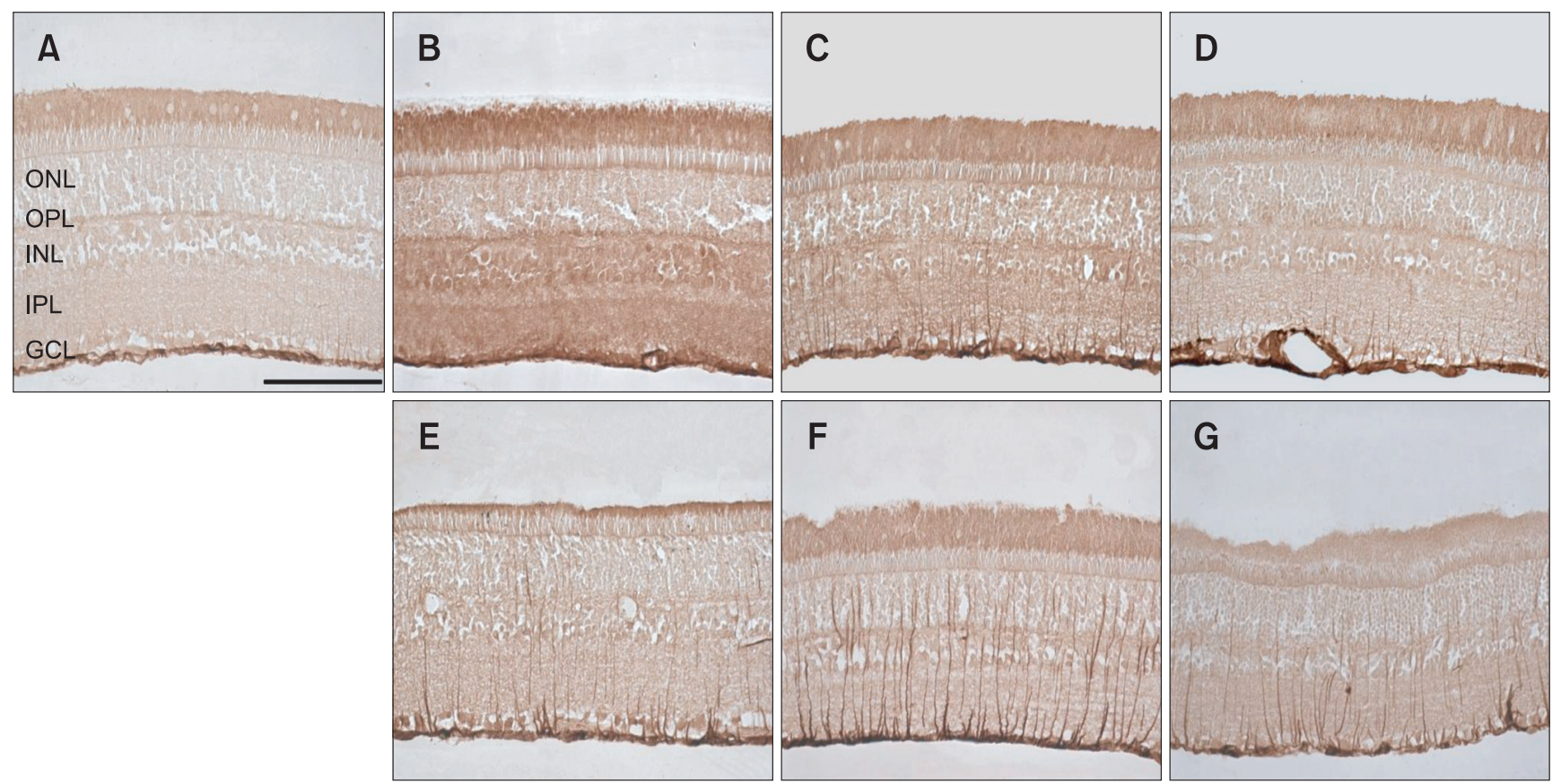

Fig. 3. Micrographs of retinal sections processed for anti-glial fibrillary acidic protein (GFAP) immunohistochemistry. GFAP immunoreactivity appears in the astrocytes and in the Müller glial end feet in the nerve fiber layer and the ganglion cell layer (GCL) of the normal (A). The GFAP immunoreactivity became stronger in both macroglial cells according to the time lapse of post venous cautery in the contralateral control retinas at 1 week (B), 4 weeks (C), and 8 weeks (D). In the glaucomatous retinas at 1 week (E), 4 weeks (F), and 8 weeks (G), strong GFAP immunoreactivity also appeared in the radial processes of the Müller glial cells throughout the whole depth of the retina. Scale bar= $50 \mu \mathrm{m}$. ONL, outer nuclear layer; OPL, outer plexiform layer; INL, inner nuclear layer; IPL, inner plexiform layer.
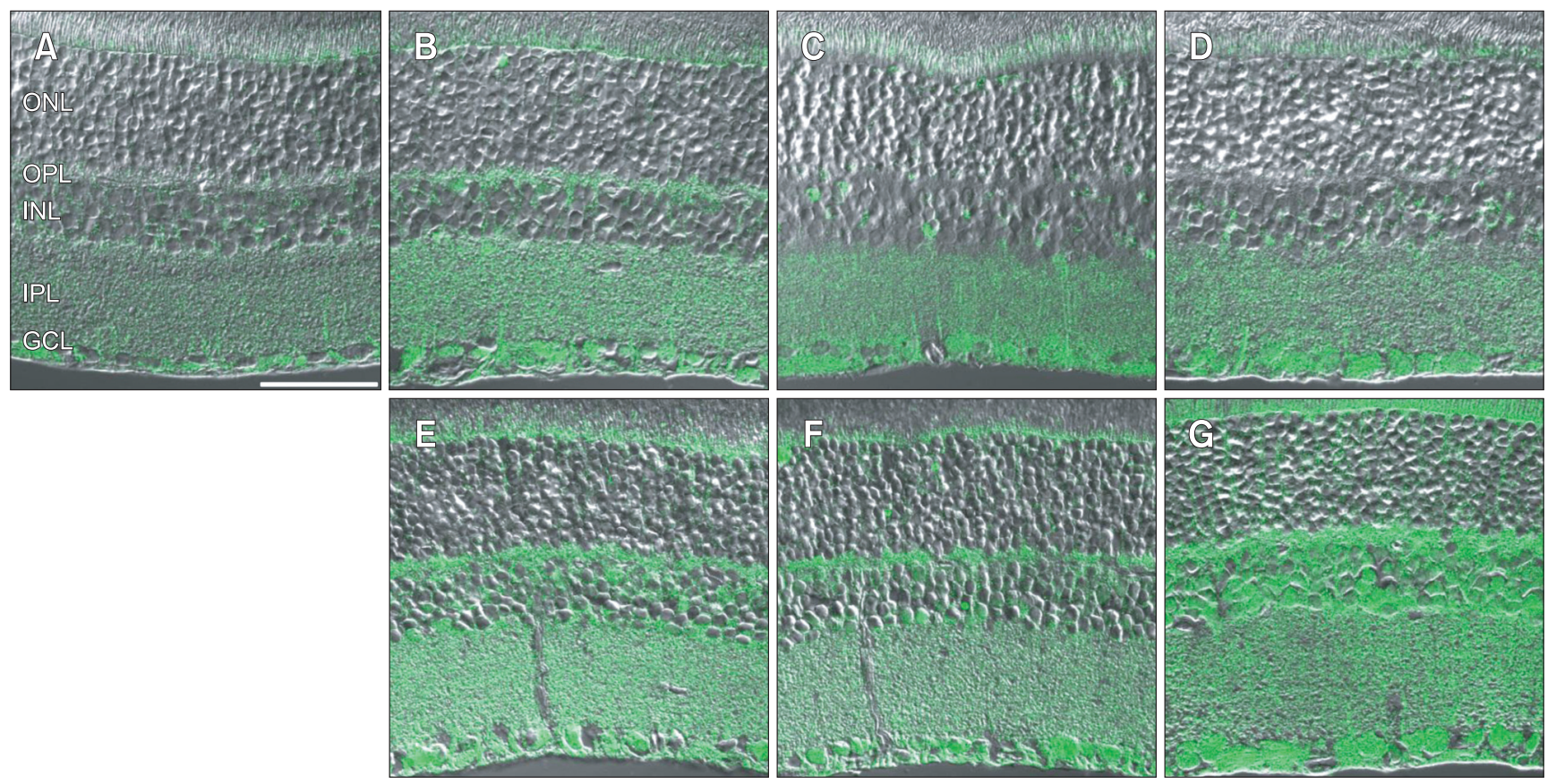

Fig. 4. Micrographs of retinal sections processed for anti-nerve growth factor (NGF) immunohistochemistry. NGF immunoreactivity appears faintly in the Müller glial end feet in the nerve fiber layer and the ganglion cell layer (GCL) of the normal (A). The NGF immunoreactivity became stronger in the end feet and proximal part of the proximal radial processes of Müller glial cells in the contralateral control retinas at 1 week (B), 4 weeks (C), and 8 weeks (D), and the reactivity in the glaucomatous retinas at 1 week (E), 4 weeks (F), and especially 8 weeks (G) was stronger than its paired contralateral control. Scale bar $=50 \mu \mathrm{m}$. ONL, outer nuclear layer; OPL, outer plexiform layer; INL, inner nuclear layer; IPL, inner plexiform layer. 

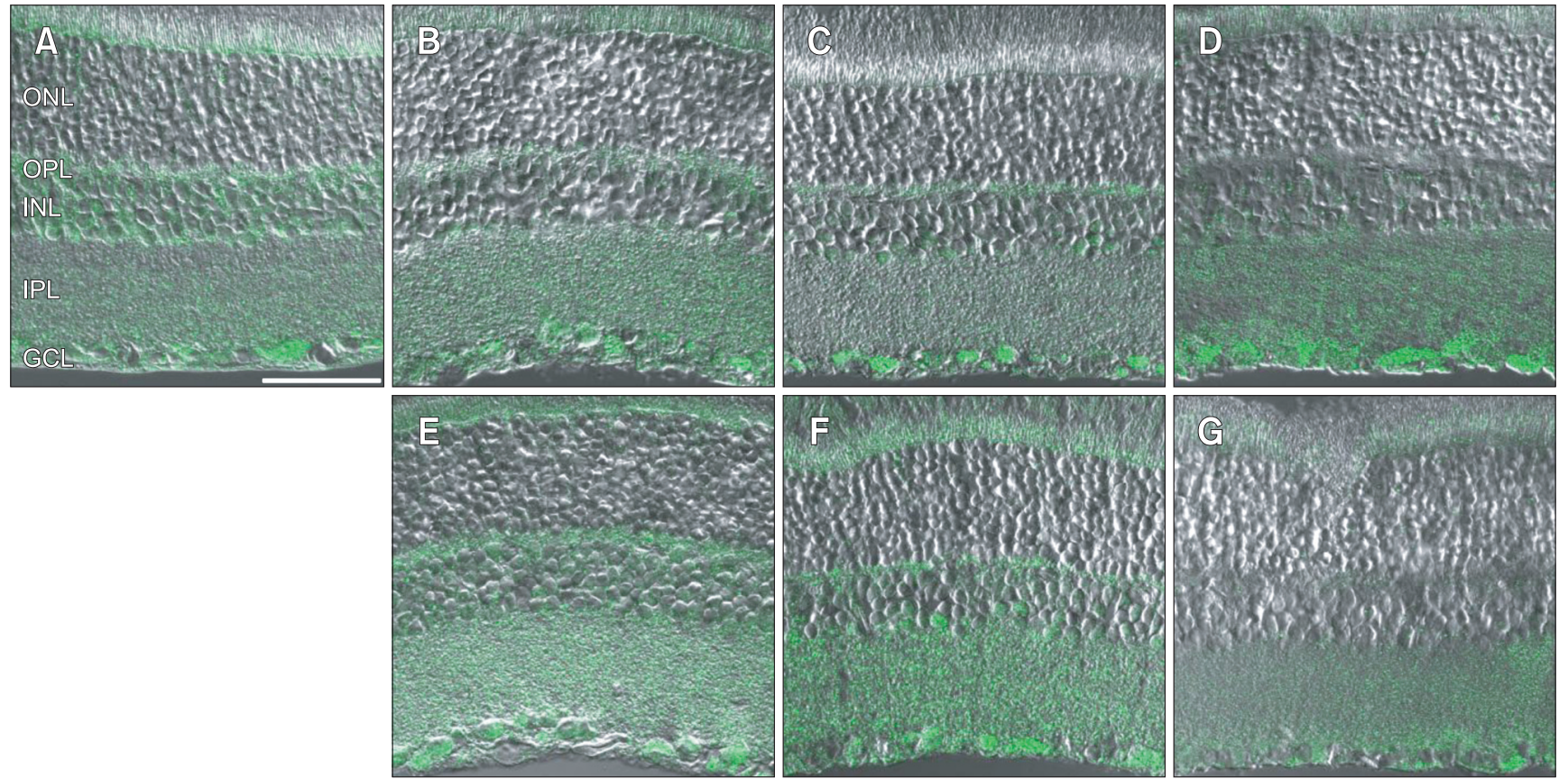

Fig. 5. Micrographs of retinal sections processed for anti-tumor necrosis factor (TNF)- $\alpha$ immunohistochemistry. TNF- $\alpha$ immunoreactivity appears faintly in the Müller glial end feet in the nerve fiber layer and the ganglion cell layer (GCL) of the normal (A). The TNF- $\alpha$ immunoreactivity became stronger in the end feet of Müller glial cells in the contralateral control retinas at 1 week (B) and at 4 weeks (C), and glaucoma retinas at 1 week (E) and at 4 weeks (F). In 8 weeks glaucoma and its control retinas (D, G), the TNF- $\alpha$ immunoreactivity appeared stronger in control (D) than the glaucoma (G). Scale bar=50 $\mu \mathrm{m}$. ONL, outer nuclear layer; OPL, outer plexiform layer; INL, inner nuclear layer; IPL, inner plexiform layer.

5F) compared to the normal. Interestingly, the reactivity at 8 weeks glaucoma was no increase (Fig. 5G). In the control retinal sections (Fig. 5B-D), the TNF- $\alpha$ reactivity was also increased in the NFL and the GCL compared to the normal.

\section{Electron Microscopic Observation}

For electron microscopic observation, the optic nerve head was taken from the eyes of the contra-lateral control to the glaucomatous and processed. In the optic nerve head, the astrocytes were distributed in between nerve fiber bundles (Fig. 6A) or near to blood vessels (Fig. 6B). They have an electron-lucent nucleus and electron- lucent pale cytoplasm, and were interconnected with their own and neighbor astrocyte cytoplasmic processes via an intercellular communicating junction, the gap junction (Fig. 6B). During glaucomatous duration lapse, the astrocytes in the paired control optic nerve head represented degenerative changes showing increase in the electron density in the nucleus and cytoplasm (Fig. 6C and D).

\section{DISCUSSION}

The present study demonstrates that retinal glial responses to glaucomatous injury happen differentially according to types of the glial cells, that is, the macroglial responses occur bilaterally in parallel to time lapse and the microglial response occurs unilaterally in the glaucomatous. Activated microglia in the ipsilateral retinas may be involved in NGF production by activated macroglial cells instead of a cytokine, TNF- $\alpha$ for neuroprotection in response to hypertensive glaucomatous injury.

Astrocytes in the glaucomatous retinas react to upregulate GFAP mRNA and protein (Inman \& Horner, 2007). While the finding of ours is in accordance with this, it is interesting that astrocytes in the contra-lateral control retinas also showed upregulated expression of GFAP protein (Kanamori et al., 2005; Ramírez et al., 2010). That might be suggested that the astrocytes distributed in the bilateral globes including the optic nerve heads and optic nerves are interconnected with each other via any connections. The assumption is proven by our electron microscopic finding with a variety of previous evidences, which report that the astrocytes are linked to each other with their cytoplasmic processes via the gap junction (Quigley, 1977; Büssow, 1980; Holländer et al., 1991; Ramírez et al., 1996). This type of intercellular communicating junction enables direct transport of small molecules such as ion, cyclic AMP and amino acids between the neighbor astrocytes. A major excitatory neurotransmitter, glutamate evokes large calcium ion increases in astrocytes (Schipke \& Kettenmann, 2004). Combined this functional aspect with our morphological alteration findings, it would be easily inferred that excessive glutamate level evoked by glaucomatous injury 

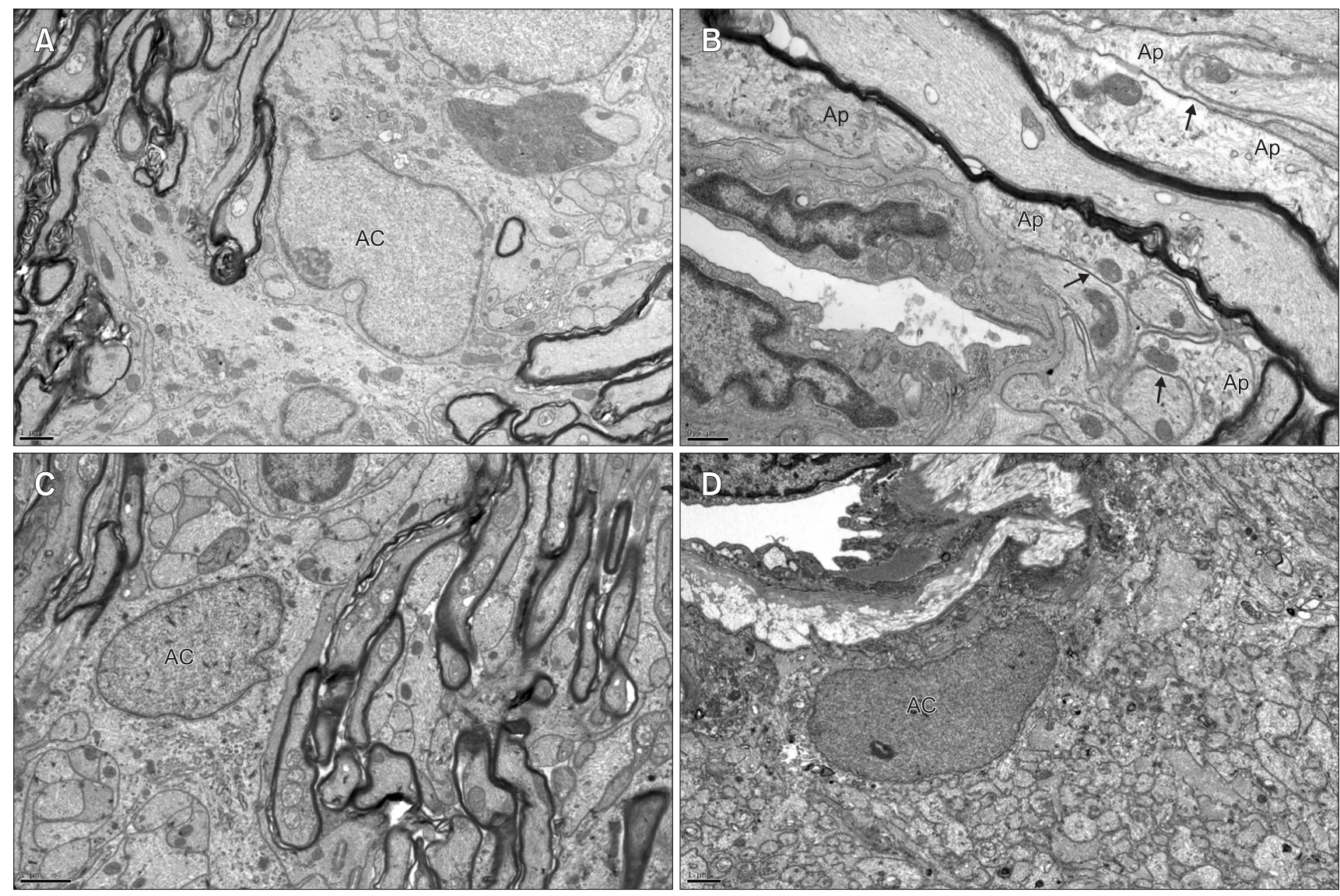

Fig. 6. Electron microscopic views of the optic nerve head in glaucoma controls. (A, B) Control of 1 week glaucoma. (C) Control of 4 weeks glaucoma. (D) Control of 8 weeks of glaucoma. Astrocytes (AC) in the optic nerve head are interposed in the myelinated nerve fiber bundles (A, C) or located close to the blood vessel (B, D). Cytoplasmic processes of astrocytes (Ap) are interconnected with the intercellular gap junction (arrows) (B). (A, C, D) Scale bars=1 $\mu \mathrm{m}$. (B) Scale bar $=0.5 \mu \mathrm{m}$.

stimulates astrocytes in the retina and optic nerve head, and consequent astroglial reaction propagates into the opposite control retina passing through the optic chiasm, optic nerve and optic nerve head via a physical link. This hypothesis also could be explained by the distribution pattern and origin of the astrocytes in the retina (Schnitzer, 1988; Ling et al., 1989; Holländer et al., 1991; Huxlin et al., 1992).

The principal glial cells in the retina, Müller cells are considered specialized astrocytes restricted only into the retina, spanning the entire thickness of the tissue and ensheath all retinal neurons. They normally expressed GFAP in the end feet, basal abutting structure of these cells on the inner limiting membrane. Thus, Müller glial reaction profiling with GFAP expressed proximal and distal radial processes responding to glaucomatous injury occurs in the ipsilateral retina. In contrast, Müller glial reaction detected by GFAP reactivity in the contralateral control retina appeared mainly in the NFL and GCL. Although it is suggested to be of involvement of immunological alterations (Kanamori et al., 2005), we consider that this event may be caused by adhering junction between astrocytes, between Müller cells, and between astrocytes and Müller cells near blood vessels and near the ganglion cell axons in the NFL and GCL, as reported by Holländer et al. (1991). That is, glial reaction of astrocytes in the glaucomatous spreads into astrocytes in the NFL and GCL of the control, and consequently transmits into the end feet of Müller cells via adherent junction.

The morphological characteristics of Müller cells allow play crucial roles in supporting neuronal function such as metabolic symbiosis, visual information processing, and the maintenance of the homeostasis of retinal extracellular milieu (Bringmann et al., 2006). It is well known that Müller cells release neurotrophic factors, uptake and degrade the excitotoxin, glutamate, and secrete the antioxidant, glutathione in response to stimuli to protect neurons (Bringmann et al., 2009). Among numerous neuroprotective factors produced by macroglial cells under pathological conditions, NGF and two types of receptors are expressed by Müller cells, and thereby these cells regulate signaling mechanism through autocrine or paracrine method (Chakrabarti et al., 1990; Hu et al., 1998; 
Wexler et al., 1998).

NGF controls development, survival, and even apoptosis of neuronal cells via two types of receptors. One is tropomyosin receptor kinase (Trk) family of high-affinity tyrosine kinase receptors which transmit prosurvival signals, and the other is the low-affinity $\mathrm{p} 75^{\mathrm{NTR}}$ receptor, a member of the TNF family which transmits anti-survival signals (Casaccia-Bonnefil et al., 1999). On the pathologic involvement of NGF, Coassin et al. (2008) report that the retinal ganglion cell death is accompanied by a timely-increase of retinal NGF and $\mathrm{p} 75^{\mathrm{NTR}}$ receptor in a rat model of glaucoma, and they conclude NGF failed trophic support due to association with anti-survival signaling receptor. Although $\mathrm{p} 75^{\mathrm{NTR}}$ level does not checked in our study, a timely increase of NGF in both retinas and a peak increase in the latest glaucomatous retina appeared.

TNF- $\alpha$ expression in our study is decreased only in the latest glaucomatous. TNF- $\alpha$ is generally considered as a key cytokine in regulating neuroinflammation, produced primarily by activated macrophages and expressed as a transmembrane protein. It can be cleaved into soluble forms to be released. Aloisi (2005) has been documented that TNF- $\alpha$ released by activated microglia makes astrocyte to produce neuroprotective factors including NGF for neuroprotection and tissue repair. We observed in this study that activation of microglia occurs not in the contralateral but in the glaucomatous eye, in accordance with the report of de Hoz et al. (2013). On the other hand, it has been described that TNF- $\alpha$ immunoreactivity is localized to the Müller cells in ischemia-reperfusion injury model (Gesslein et al., 2010). Taken all together, our study seems to be suggested that activated microglia releases soluble form of TNF- $\alpha$, and this cytokine in turn stimulates macroglial cells to produce neuroprotective factors in response to a relatively mild-hypertensive glaucomatous injury compared to the experimental condition of Coassin et al. (2008). Furthermore, a reciprocal expression of NGF and TNF- $\alpha$ only in the latest glaucomatous retina may suggest that these relationships collaborate not for neurodegeneration but for neuroprotection.

\section{SUMMARY}

GFAP immunoreactivity appeared in the astrocytes and end feet of Müller cells in the normal retinas. Whereas glial reaction of the astrocytes occurs in both retinas spreading via the gap junction, Müller glial reaction is happened profiling the whole cell in the glaucomatous retina. Microglial cells increase in reactivity and in cell number along the time course in the glaucomatous. NGF is expressed a timely increase in both retinas recording a peak in the latest glaucomatous, while TNF- $\alpha$ is also expressed with the same manner except the latest glaucomatous showing no reactivity. TNF- $\alpha$ produced by activated microglia may serve for NGF production by activated macroglial cells in response to this hypertensive glaucomatous injury.

\section{CONFLICT OF INTEREST}

No potential conflict of interest relevant to this article was reported.

\section{REFERENCES}

Akiyama H, Nakazawa T, Shimura M, Tomita H, and Tamai M (2002) Presence of mitogen-activated protein kinase in retinal Muller cells and its neuroprotective effect ischemia-reperfusion injury. Neuroreport 13, 2103-2107.

Aloisi F (2001) Immune function of microglia. Glia 36, 165-179.

Aloisi F (2005) Cytokine production. In: Neuroglia, eds. Kettenmann H and Ransom B R, pp. 285-301, (Oxford University Press, Oxford).

Bauer H, Bauer H C, Haseloff R F, and Blasig I E (2005) The role of glia in the formation and function of the blood-brain barrier. In: Neuroglia, eds. Kettenmann H and Ransom B R, pp. 325-333, (Oxford University Press, Oxford).

Bignami A and Dahl D (1979) The radial glia of Müller in the rat retina and their response to injury. An immunofluorescence study with antibodies to glial fibrillary acidic (GFA) protein. Exp. Eye Res. 28, 6369.

Bringmann A, landiev I, Pannicke T, Wurm A, Holbron M, Wiedmann P, Osborne N N, and Reichenbach A (2009) Cellular signaling and factors involved in Müler cell gliosis: neuroprotective and detrimental effects. Prog. Retin. Eye Res. 28, 423-451.

Bringmann A, Pannicke T, Grosche J, Francke M, Wiedemann P, Skatchkov S N, Osborne N N, and Reichenbach A (2006) Müller cells in the healthy and diseased retina. Prog. Retin. Eye Res. 25, 397424.

Bringmann A and Reichenbach A (2001) Role of Müller cells in retinal degenerations. Front. Biosci. 6, E77-E92.

Büssow H (1980) The astrocytes in the retina and optic nerve head of mammals: a special glia for the ganglion cell axons. Cell Tissue Res. 206, 367-378.

Casaccia-Bonnefil P, Gu C, and Chao M V (1999) Neurotrophins in cell survival/death decisions. Adv. Exp. Med. Biol. 468, 275-282.

Chakrabarti S, Sima A A, Lee J, Brachet P, and Dicou E (1990) Nerve growth factor (NGF), proNGF and NGF receptor-like immunoreactivity in $\mathrm{BB}$ rat retina. Brain Res. 523, 11-15.

Coassin M, Lambiase A, Sposato V, Micera A, Bonini S, and Aloe L (2008) Retinal p75 and bax overexpression is associated with retinal 
ganglion cells apoptosis in a rat model of glaucoma. Graefes Arch Clin. Exp. Ophthalmol. 246, 1743-1749.

de Hoz R, Gallego B I, Ramírez A I, Rojas B, Salazar J J, Valiente-Soriano F J, Avilés-Trigueros M, Villegas-Perez M P, Vidal-Sanz M, Triviño A, and Ramírez J M (2013) Rod-like microglia are restricted to eyes with laser-induced ocular hypertension but absent from the microglial changes in the contralateral untreated eye. PLoS One 8, e83733.

Delgado M, Leceta J, and Ganea D (2003) Vasoactive intestinal peptide and pituitary adenylate cyclase-activating polypeptide inhibit the production of inflammatory mediators by activated microglia. $J$. Leukocyte Biol. 73, 155-164.

Eichler W, Yafai Y, Wiedemann P, and Reichenbach A (2004) Angiogenesis-related factors derived from retinal glial (Muller) cells in hypoxia. Neuroreport 15, 1633-1637.

Francke M, Pannicke T, Biedermann B, Faude F, Wiedemann P, Reichenbach A, and Reichelt W (1997) Loss of inwardly rectifying potassium currents by human retinal glial cells in diseases of the eyes. Glia 20, 210-218.

Gesslein B, Håkanson G, Gustafsson L, Ekström P, and Malmsjö M (2010) Tumor necrosis factor and its receptors in the neuroretina and retinal vasculature after ischemia-reperfusion injury in the pig retina. Mol. Vis. 16, 2317-2327.

Hammes H P, Federoff H J, and Brownlee M (1995) Nerve growth factor prevents both neuroretinal programmed cell death and capillary pathology in experimental diabetes. Mol. Med. 1, 527-534.

Holländer H, Makarov F, Dreher Z, van Driel D, Chang-Ling T L, and Stone $J$ (1991) Structure of the macroglia of the retina: sharing and division of labour between astrocytes and Müller cells. J. Comp. Neurol. 313, 587-603.

Hsu S M, Raine L, and Fanger H (1981) Use of avidin-biotin-peroxidase complex $(\mathrm{ABC})$ in immunoperoxidase techniques. J. Histochem. Cytochem. 29, 577-580.

Hu B, Yip H K, and So K F (1998) Localization of p75 neurotrophin receptor in the retina of the adult SD rat: an immunocytochemical study at light and electron microscopic levels. Glia 24, 187-197.

Huxlin K R, Sefton A J, and Furby J H (1992) The origin and development of retinal astrocytes in the mouse. J. Comp. Neurol. 21, 530-544.

Inman D M and Horner P J (2007) Reactive nonproliferative gliosis predominates in a chronic mouse model of glaucoma. Glia 55, 942 953.

Kanamori A, Nakamura M, Nakanishi Y, Yamada Y, and Negi A (2005) Long-term glial reactivity in rat retinas ipsilateral and contralateral to experimental glaucoma. Exp. Eye Res. 81, 48-56.

Lee J I, Shin J M, Yeum C E, Chae G T, Chun M H, and Oh S J (2012) Intravitreal delivery of mesenchymal stem cells loaded onto hydrogel affects the regulatory expression of endogenous NGF and BDNF in ischemic rat retina. Tissue Eng. Reg. Med. 9, 249-258.

Ling T, Mitrofanis J, and Stone J (1989) Origin of retinal astrocytes in the rat: evidence of migration from the optic nerve. J. Comp. Neurol. 286,
345-352.

Pannicke T, landiev I, Uckermann O, Biedermann B, Kuzera F, Wiedemann P, Wolburg H, Reichenbach A, and Bringmann A (2004) A potassium channel-linked mechanism of glial cell swelling in the postischemic retina. Mol. Cell Neurosci. 26, 493-502.

Quigley H A (1977) Gap junctions between optic nerve head astrocytes. Invest. Ophthalmol. Vis. Sci. 16, 582-585.

Quigley H A and Broman A T (2006) The number of people with glaucoma worldwide in 2010 and 2020. Br. J. Ophthalmol. 90, 262-267.

Quigley H A, Nickells R W, Kerrigan L A, Pease M E, Thibault D J, and Zack D J (1995) Retinal ganglion cell death in experimental glaucoma and after axotomy occurs by apoptosis. Invest. Ophthalmol. Vis. Sci. 36, 774-786.

Ramírez A I, Salazar J J, de Hoz R, Rojas B, Gallego B I, Salinas-Navarro M, Alarcón-Martínez L, Ortín-Martínez A, Avilés-Trigueros M, Vidal-Sanz M, Triviño A, and Ramírez J M (2010) Quantification of the effect of different levels of IOP in the astroglia of the rat retina ipsilateral and contralateral to experimental glaucoma. Invest. Ophthalmol. Vis. Sci. 51, 5690-5696.

Ramírez J M, Triviño A, Ramírez A I, Salazar J J, and Garcia-Sanchez J (1996) Structural specializations of human retinal glial cells. Vision Res. 36, 2029-2036.

Rodrieck R W (1998) The First Steps in Seeing. pp. 7-34, (Sinauer Associates Inc., Sunderland).

Rungger-Brändle E, Dosso A A, and Leuenberger P M (2000) Glial reactivity, an early feature of diabetic retinopathy. Invest. Ophthalmol. Vis. Sci. 41, 1971-1980.

Schipke C G and Kettenmann H (2004) Astrocyte responses to neuronal activity. Glia 47, 226-232.

Schnitzer J (1988) Astrocytes in the guinea pig, horse, and monkey retina: their occurrence coincides with the presence of blood vessels. Glia 1, 74-89.

Stone J and Dreher Z (1987) Relationship between astrocytes, ganglion cells and vasculature of the retina. J. Comp. Neurol. 255, 35-49.

Swanson R A (2005) Astrocyte neurotransmitter uptake. In: Neuroglia, eds. Kettenmann H and Ransom B R, pp. 346-354, (Oxford University Press, Oxford).

Tezel G, Chauhan B C, LeBlanc R P, and Wax M B (2003) Immunohistochemical assessment of the glial mitogen-activated protein kinase activation in glaucoma. Invest. Ophthalmol. Vis. Sci. 44, 3025-3033.

Varma R, Ying-Lai M, Francis B A, Nguyen B B T, Deneen J, Wilson M R, Azen S P, and Los Angeles Latino Eye Study Group (2004) Prevalence of open-angle glaucoma and ocular hypertension in Latinos: the Los Angeles Latino Eye Study. Ophthalmology 111, 1439-1448.

Wexler E M, Berkovich O, and Nawy S (1998) Role of the low-affinity NGF receptor (p75) in survival of retinal bipolar cells. Vis. Neurosci. 15, 211-218. 